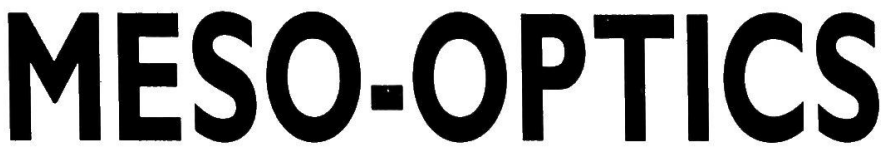

Foundations and Applications 
Cover:

Design of the meso-optical mirror with ring response (a half) which was fabricated in 1986 with diffraction limited resolution. 


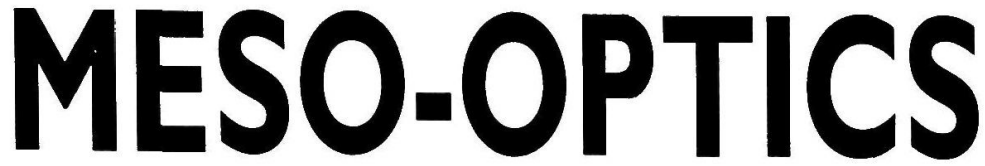

\section{Foundations and Applications}

\section{M Soroko}

foint Institute for Nulcear Research, Russia 


\section{Published by}

World Scientific Publishing Co. Pte. Ltd.

P O Box 128, Farrer Road, Singapore 912805

USA office: Suite 1B, 1060 Main Street, River Edge, NJ 07661

UK office: 57 Shelton Street, Covent Garden, London WC2H 9HE

\section{British Library Cataloguing-in-Publication Data}

A catalogue record for this book is available from the British Library.

\section{MESO-OPTICS - FOUNDATIONS AND APPLICATIONS}

Copyright (C) 1996 by World Scientific Publishing Co. Pte. Ltd.

All rights reserved. This book, or parts thereof, may not be reproduced in any form or by any means, electronic or mechanical, including photocopying, recording or any information storage and retrieval system now known or to be invented, without written permission from the Publisher.

For photocopying of material in this volume, please pay a copying fee through the Copyright Clearance Center, Inc., 222 Rosewood Drive, Danvers, MA 01923, USA. In this case permission to photocopy is not required from the publisher.

ISBN 981-02-2700-0 
In memory

of my wife,

Valentina M. Soroko

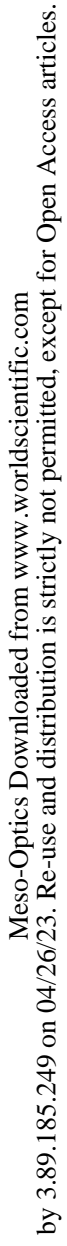


This page is intentionally left blank 


\section{Abstract}

"ALMOST EVERYTHING ABOUT CONICAL WAVEFRONTS" is the motto of this book, which can be conceived as an up-to-date encyclopedic review of the theoretical and experimental investigations, performed by many researchers in the field of meso-optics. With many examples it is illustrated that meso-optics is indeed a rapidly developing branch of modern optics, in which the conical wavefronts are used, though sometimes implicitly, to transfer in space the information about the objects or the electromagnetic energy.

By starting from axicons, various meso-optical elements and new scopes of meso-optics is described. Diffraction free light beams and the localized electromagnetic wavefields is discussed. It is shown that gravitational lens can be accepted as a beamsplitter in the interferometer of the cosmic scale. Of particular interest will be inverse meso-optics, which can be used in electron accelerators for transportation of synchrotron radiation and in micromechanics. Some meso-optical microscopes and meso-optical metrological devices will be suggested. The history of meso-optics is followed from the first gravitational lenses in our universe up to the localized electromagnetic fields developed recently. Some integral transforms, useful in meso-optics, is presented in the Appendix. 
This page is intentionally left blank 


\section{PREFACE}

The aim of this book is to acquaint the reader with up-to-date theoretical, experimental and instrumental contributions of many researchers to the new branch of modern optics which is referred to as "meso-optics".

The theoretical contribution follows from the new insight into the traditional optical imaging systems, such as lens, which focuses the light along two orthogonal directions. It has been discovered that imaging systems with axial symmetry, which focus the light only along one coordinate and not along another, orthogonal coordinate, can be used effectively for solving some awkward or even deadlock problems. In the course of more than ten years we have been using the term "meso-optics" to say that conical wavefronts do contribute principally into the systems for transferring in space the information about the object and also for transmitting the electromagnetic energy at long distances.

The experimental contribution covers the observations of various new phenomena with conical wavefronts, such as longitudinal interference of the diffraction free wavefronts, the decisive demonstration that information about the position of the microscopic straight-like objects in $3 \mathrm{D}$ space can be converted into two point-like spots with high data compression by means of sophisticated meso-optical imaging system, and the evident description of the exotic phenomenon, "darkness" in optics and meso-optics.

The instrumental contribution covers traditional and nontraditional techniques in optics, holography and tomography, which seems to be the most adequate to meso-optics. We have now diamond turning technique, computer generated hologram, precise plotter for manufacturing kinoform with prescribed phase relief and the technique for making "focusators". The first kinoform for visible light with diffraction limited resolution was fabricated in 1984. The first precise meso-optical mirror with ring response was made in 1986. Therefore any proposals presented in this book in the field of meso-optics, for visible light and X-ray radiation, are provided with adequate technology basis.

In addition to meso-optics, by means of which the object point is transformed into the line segment or circle, "inverse meso-optics", which enables one to convert into the point of coherent radiation generated by the source in the form of a straight line segment was also discovered. The direct application of inverse meso-optics for transportation of the $\mathrm{X}$-ray synchrotron radiation and for manufacture of micromechanical parts of the devices is well evident. 
What else can we benefit from meso-optics? First of all it must be stated, that the problem of the depth of focus in microscopy was solved by means of the meso-optical Fourier transform microscope, in which the information about position of the straight-like microscopic object has been compressed up to two point-like spots of the diffraction limited dimensions. The new device, confocal meso-optical scanning microscope is to be noticed. All meso-optical microscopes and tomographs, suggested in this book, have no counterparts in the conventional optical microscopy and tomography. These systems offer high productivity and can be applied adequately in microscopic robotics vision, for material testing in industry and in high energy particle physics.

This book is organized as follows. After introduction to meso-optics, the properties of conical wavefronts are explained in detail, and some applications. of axicons are described. Meso-optical elements with ring response and their adequate application in the meso-optical Fourier transform microscope is also explained. The theory of this device and its features are presented along with description of some other designs of this device and application of the mesooptical Fourier transform microscope in high energy physics.

The diffraction free wavefields, Bessel-Gauss beams and various devices for generation of the diffraction free fields are described further. Following explanation of the exotic phenomenon "darkness" in optics and meso-optics, the longitudinal interference of the diffraction free wavefronts and its application in ophthalmology are presented. The problem of the localized electromagnetic waves is treated along with theoretical and experimental investigations in this rapidly developing part of meso-optics.

Gravitational lens, which is also a meso-optical element, accomplishes nonlinear transformation of the space coordinates and velocity components of the celestial objects. Typical examples of the gravitational lenses discovered recently and the property of gravitational lens as a beam-splitter is described. The fundamental properties of the critical curve (caustics) which separates the space into areas with different number of meso-optical images are considered with emphasis on the parallax gravitational effect in binary and planetary systems. The main topics of gravitational lens applications are presented.

Two chapters of this book are devoted to confocal meso-optical microscopemicrotomographs, new stereo-microscope with high depth resolution and to some meso-optical metrological devices. Some potential new applications of meso-optics in science, industry and medicine is suggested throughout this book.

The history of meso-optics, presented in this book, starts from the early stage of our universe, to first gravitational lenses, followed by publications 
in the last century and finally up-to-date innovations, many of which were proposed and performed with active participation of the author. The Appendix contains some integral transforms, which are useful in meso-optics.

The author believes that after publication of this book the frontiers of meso-optics will be expanded and its development will be accelerated.

L. M. Soroko
Dubna, Russia 
This page is intentionally left blank 


\section{Acknowledgments}

I wish to thank V. P. Dzhelepov, corresponding member of the USSR Academy of Sciences, and Director of the Laboratory of Nuclear Problems, Joint Institute for Nuclear Research (Dubna), for support of investigations in meso-optics. Gratitude is also extended to Professor S. A. Bunyatov, Chief of the project Neutrino Detector, and to Professor Yu. A. Batusov for their support and discussions. The development of the meso-optical Fourier transform microscope was accomplished as part of the USSR-Hungary scientific collaboration, and I am grateful to Professor D. Kiss (KFKI Budapest), Chief of the Hungarian scientific group. Warm appreciation is expressed to Gy. L. Bencze, A. Kishvaradi, L. Molnar, G. Nitrai and J. Molnar, as well as to V. V. Tereshchenko. I wish to acknowledge A. Ya. Astakhov, V. I. Prichod'ko, V. A. Drozdov, A. L. Men'shikov, A. V. Nikul'nikov from the Laboratory of Computing Techniques and Automation of JINR, V. P. Koronkevich and I. G. Paltchikova from the Institute of Automation and Electrometry in Novosibirsk for their collaboration.

I must thank Professor Ya. A. Smorodinsky from the Laboratory of Theoretical Physics, of JINR for many discussions, in particular on diffraction free waves, symplectic groups and gravitational lens.

Finally I wish to thank L. Gavrilenko and N. Tikhomirova for excellent typing, G. V. Gorbunova for high quality photocopies and V. I. Krivozoubova for her continuous support and patience. 
This page is intentionally left blank 
This page is intentionally left blank 


\section{MESOOPTICS $(\sqrt{i})$ MESOOPTICS}

Symbol of Meso-Optics. 
This page is intentionally left blank 


\section{CONTENTS}

Abstract . . . . . . . . . . . . . . . . . . . . vii vii

Preface . . . . . . . . . . . . . . . . . . ix

Acknowledgments . . . . . . . . . . . . . . . . . . . . xiii

Chapter 1. Introduction and Survey . . . . . . . . . . . . . 5

Chapter 2. Axicons and Conical Waves . . . . . . . . . . . 5

2.1. Conical waves and meso-optics . . . . . . . . . . . . . . . 5

2.2. Axicons . . . . . . . . . . . . . . . . . . . . . . . . . 8

2.3. Side-lobes of the meso-optical image . . . . . . . . . . . . . 21

2.4. General theory of axicons . . . . . . . . . . . . . . . . . . 24

2.5. Computer-generated holograms . . . . . . . . . . . . . . . 28

2.6. Kinoforms and focusators . . . . . . . . . . . . . . . . . . 29

2.7. Description of the conical waves . . . . . . . . . . . . . . . 31

2.8. Exotic meso-optical elements . . . . . . . . . . . . . . . . 33

2.8.1. Right-angle reflaxicon . . . . . . . . . . . . . . . . . 33

2.8.2. Axicon with annular aperture . . . . . . . . . . . . . 35

2.8.3. Axial non-symmetrical meso-optical elements . . . . . 36

2.9. Applications of axicons . . . . . . . . . . . . . . . . . . . 40

2.9.1. Axiconic laser string . . . . . . . . . . . . . . . . . 40

2.9.2. A pair of convergent and divergent axicons . . . . . . . 41

2.9.3. Conoid axicon . . . . . . . . . . . . . . . . . . . . 41

2.9.4. Light sectioning with axicon . . . . . . . . . . . . . . 42

2.9.5. Axicon-scanned Fabry-Perot spectrometer . . . . . . . . 42

2.9.6. Aberration free axicon-objective . . . . . . . . . . . . 44

2.9.7. Axicon in the X-ray laser . . . . . . . . . . . . . . . 45

Chapter 3. Meso-Optical Elements with Ring Response . . . 49

3.1. Introduction . . . . . . . . . . . . . . . . . . . . . . . 49

3.2. Meso-optical Fourier Transform Microscope (MFTM) . . . . . . 50

3.3. Sagittal section of the MFTM . . . . . . . . . . . . . . . . 62

3.4. Convolution kernel of the MFTM . . . . . . . . . . . . . . 64

3.5. Meso-optical moiré-effect . . . . . . . . . . . . . . . . . . 67 
3.6. MFTM with one-channel photodetectors . . . . . . . . . . . 73

3.7. Some other designs of the MFTM . . . . . . . . . . . . . . 81

3.7.1. FT-microscope of the direct observation . . . . . . . . . 81

3.7.2. Acceptance of the FT-microscopes . . . . . . . . . . . 83

3.7.3. MFTM with double focusing . . . . . . . . . . . . . . . 91

3.8. Isoplanatic systems in meso-optics . . . . . . . . . . . . . . 102

3.9. Problem of depth-of-focus in meso-optics . . . . . . . . . . . 115

3.10. Another systems of the line detection . . . . . . . . . . . . . 118

3.11. Applications . . . . . . . . . . . . . . . . . . . . . . 122

Chapter 4. Diffraction Free Wavefields . . . . . . . . . . . . 127

4.1. Introduction . . . . . . . . . . . . . . . . . . . . . . . 127

4.2. Bessel-Gauss beams . . . . . . . . . . . . . . . . . . . . 131

4.3. Darkness in optics . . . . . . . . . . . . . . . . . . . . . 135

4.4. Darkness in meso-optics . . . . . . . . . . . . . . . . . . 138

4.5. Longitudinal interference of the conical wavefields . . . . . . . 146

4.6. Production of diffraction-free waves . . . . . . . . . . . . . 159

Chapter 5. Localized Electromagnetic Waves . . . . . . . 165

5.1. Introduction . . . . . . . . . . . . . . . . . . . . . . . 165

5.2. Light beams and complex-source point method . . . . . . . . 166

5.3. Focus wave modes . . . . . . . . . . . . . . . . . . . . . 169

5.4. Bidirectional representation . . . . . . . . . . . . . . . . . 174

5.5. Localized Gaussian fields . . . . . . . . . . . . . . . . . . 176

5.6. Localized Bessel-Gauss fields . . . . . . . . . . . . . . . . 177

5.7. Experimental verification . . . . . . . . . . . . . . . . . . 179

5.8. Localized waves for the Klein-Gordon equation . . . . . . . . 183

5.9. Approximate focus wave modes . . . . . . . . . . . . . . . 184

5.10. Electromagnetic "missiles" and "bullets" . . . . . . . . . . . 186

Chapter 6. Gravitational Lens . . . . . . . . . . . . . . . . 187

6.1. Introduction . . . . . . . . . . . . . . . . . . . . . . 187

6.2. General theory . . . . . . . . . . . . . . . . . . . 187

6.3. Moving gravitational lens . . . . . . . . . . . . . . . . . . 197

6.4. Examples of the gravitational lens . . . . . . . . . . . . . . 200

6.5. Gravitational lens as a beamsplitter . . . . . . . . . . . . . 201

6.6. Gravitational micro-lensing . . . . . . . . . . . . . . . . . 207 
6.7. Caustics . . . . . . . . . . . . . . . . . . . . . . 209

6.8. Parallax effect . . . . . . . . . . . . . . . . . . . . . . 215

6.9. Optical depth . . . . . . . . . . . . . . . . . . . . . . . 218

6.10. Binaries and planetary systems . . . . . . . . . . . . . . . 222

6.11. Applications . . . . . . . . . . . . . . . . . . . . . . . 226

6.11.1. Problem of the dark halo objects . . . . . . . . . . . 226

6.11.2. Giant luminous arcs . . . . . . . . . . . . . . . . . 229

6.11.3. Large scale inhomogeneities of the universe . . . . . . . 231

6.11.4. The Hubble's parameter . . . . . . . . . . . . . . . 232

Chapter 7. Inverse Meso-Optics . . . . . . . . . . . . . . . 235

7.1. Introduction . . . . . . . . . . . . . . . . . . . 235

7.2. Undulator radiation . . . . . . . . . . . . . . . . . . . . 238

7.3. Meso-optical undulator . . . . . . . . . . . . . . . . . . . 242

7.4. Self-focusing undulator-wiggler . . . . . . . . . . . . . . . 245

7.5. Undulator with apodized spectral lines . . . . . . . . . . . . 246

7.6. Interference of synchrotron radiation . . . . . . . . . . . . . 247

7.7. Meso-optical Cherenkov gas counter . . . . . . . . . . . . . 250

Chapter 8. Meso-Optics and Micro-Tomography . . . . . . . 259

8.1. Modern microscopes for 3D viewing . . . . . . . . . . . . . 259

8.2. Methods of light sectioning . . . . . . . . . . . . . . . . . 262

8.3. Confocal scanning microscope . . . . . . . . . . . . . . . . 264

8.4. Microscope with rotating oblique illumination . . . . . . . . . 269

8.5. Meso-optical microscopes as tomographic devices . . . . . . . 270

8.6. Meso-optical confocal microscopes . . . . . . . . . . . . . . 276

8.7. New stereo-microscope . . . . . . . . . . . . . . . . . . . 277

Chapter 9. Meso-Optical Metrological Devices . . . . . . . . 281

9.1. Traditional testing of the conical surfaces . . . . . . . . . . . 281

9.2. Meso-optical conicometers . . . . . . . . . . . . . . . . . 283

9.3. Meso-optical interferometer . . . . . . . . . . . . . . . . . 286

9.4. Meso-optical profilometers . . . . . . . . . . . . . . . . . 287

9.5. Meso-optical keratometer . . . . . . . . . . . . . . . . . . 288

9.6. Informational content of the high order diffraction pattern . . . 295

9.7. Meso-optical microscope of high productivity for vertical particle tracks 
9.8. Caustic meso-optical confocal microscope for vertical particle tracks . . . . . . . . . . . . . . . . . . . . . 307

Chapter 10. History of Meso-Optics . . . . . . . . . . . . . 311

10.1. From Big Bang to the gravitational lens . . . . . . . . . . . 311

10.2. Ancient history - Middle ages - XX century . . . . . . . . . 311

10.3. Diastoloscope . . . . . . . . . . . . . . . . . . . . . . . 312

10.4. Theory of the gravitational lens . . . . . . . . . . . . . . . 313

10.5. Meso-optics . . . . . . . . . . . . . . . . . . . . . . . 316

10.6. Diffraction free wavefields . . . . . . . . . . . . . . . . . . 317

10.7. Localized wavefields . . . . . . . . . . . . . . . . . . . 318

Appendix: Integral Transforms . . . . . . . . . . . . . . . 319

A.1. Fourier transform . . . . . . . . . . . . . . . . . . . . . 319

A.2. Hartley transform . . . . . . . . . . . . . . . . . . . . . 320

A.3. Hilbert transform . . . . . . . . . . . . . . . . . . . . . 322

A.4. Abbe transform . . . . . . . . . . . . . . . . . . . . . . 323

References .. . . . . . . . . . . . . . . . 329

Subject Index . . . . . . . . . . . . . . . . . . . . . . . . . 359

Author Index . . . . . . . . . . . . . . . . . . . . . 403 